\title{
Additive effects of atezolizumab and bevacizumab plus chemotherapy for patients with non-small cell lung cancer regardless of presence of EGFR mutations, ALK rearrangements, or PD-L1 expression
}

\author{
Takehiro Uemura, Toyoaki Hida \\ Department of Thoracic Oncology, Aichi Cancer Center Hospital, Nagoya, Aichi, Japan \\ Correspondence to: Toyoaki Hida, MD, PhD. Department of Thoracic Oncology, Aichi Cancer Center Hospital, 1-1 Kanokoden, Chikusa-ku, Nagoya, \\ Aichi 464-8681, Japan. Email: 107974@aichi-cc.jp. \\ Comment on: Socinski MA, Jotte RM, Cappuzzo F, et al. Atezolizumab for First-Line Treatment of Metastatic Nonsquamous NSCLC. N Engl J Med \\ 2018;378:2288-301.
}

Submitted Jul 30, 2018. Accepted for publication Aug 08, 2018.

doi: $10.21037 /$ tcr.2018.08.14

View this article at: http://dx.doi.org/10.21037/tcr.2018.08.14

The first-line treatment protocol for advanced nonsmall cell lung cancer (NSCLC) has improved with immune checkpoint inhibitor (ICI) development. The Food and Drug Administration (FDA) has approved three ICIs for second-line treatment of NSCLC (nivolumab, pembrolizumab, and atezolizumab), and randomized studies have shown superior overall survival (OS) with ICIs compared with second-line docetaxel (1-4). Moreover, pembrolizumab was approved as a first-line treatment because of its superior efficacy in the first-line treatment of tumors with $\mathrm{PD}-\mathrm{L} 1 \geq 50 \%$ versus chemotherapy [progression-free survival (PFS) of 10.4 versus 6.0 months, respectively] (5). Durvalumab, another ICI, also showed a longer PFS following consolidation therapy than placebo in patients with stage III NSCLC without disease progression after $\geq 2$ platinum-based chemoradiotherapy cycles (6). These advances for managing NSCLC are important, but a number of patients will not benefit from these singleagent therapies, and other strategies using ICIs are being evaluated. Increasing evidence from studies suggests that chemotherapy mediates its antitumor activity through cytotoxic effects and immunological effects, including T-regulatory cell activity reduction and cross-presentation enhancement of tumor antigens (7-9). Reportedly, chemotherapy induces PD-L1 expression on tumor cells $(10,11)$. Thus, a combination of immunotherapy and chemotherapy may synergistically improve the anticancer activity of anti-PD-1 and anti-PD-L1 monotherapy. Combinations of anti-PD-1 or anti-PD-L1 antibodies with standard chemotherapy or with anti-CTLA-4 antibody are the primary approaches that are being applied. Attempting to improve survival in NSCLC patients beyond the subgroup of highly expressing PD-L1 patients, the KEYNOTE-021 study tested a combination of pemetrexed and carboplatin for treating patients with previously untreated metastatic NSCLC (12). In this randomized, open-label, phase II study, the overall response rate (ORR) and PFS for patients randomized to the pembrolizumab plus chemotherapy group was improved and the treatmentrelated adverse events were tolerable. These results, along with the manageable safety profile led to the FDA approval of pembrolizumab, with pemetrexed and carboplatin, as first-line combination therapy. It is possible that the use of multiple agents may enhance their effectiveness in terms of increasing OS. The results of other clinical trials of combination therapies including ICIs are being published these days.

In the June 2018 issue of The New England Fournal of Medicine, Socinski and colleagues demonstrated a significant improvement in PFS and OS among patients with metastatic non-squamous NSCLC administered atezolizumab plus bevacizumab and chemotherapy (IMpower-150)—regardless of their PD-L1 expression and EGFR or ALK genetic alteration status (13). The following patients were included 
in the study: those who had stage IV or recurrent metastatic non-squamous NSCLC and were not treated with chemotherapy, those with a baseline Eastern Cooperative Oncology Group (ECOG) performance status score of 0/1, and those with any PD-L1 immunohistochemistry status. Additionally, patients with $E G F R$ or $A L K$ genomic alterations were enrolled treatment with at least one approved tyrosine kinase inhibitor had previously resulted in disease progression or unacceptable side effects. The international, open-label, phase III study enrolled a total of 1,202 patients randomly assigned to 1 of 3 groups: the atezolizumab plus carboplatin plus paclitaxel, ACP group (402 patients), the atezolizumab plus bevacizumab plus carboplatin plus paclitaxel, ABCP group (400 patients), or the bevacizumab plus carboplatin plus paclitaxel, BCP group (400 patients). Induction treatments were administered for four or six 21-day cycles. After that, patients were continually administered atezolizumab, bevacizumab, or both until unmanageable toxic effects or disease progression was observed. Patients were allowed to continue atezolizumab after disease progression in case any clinical benefits were evidenced. Atezolizumab was ensured to have no crossover.

The two primary end points were PFS, among both patients in the intention-to-treat population with a wildtype (WT) population and those in the WT population with high expression of an effector T-cell (Teff) gene signature in the tumor (Teff-high WT population), and OS in the WT population. The Teff gene signature was described as PD-L1, CXCL9, and IFN- $\gamma$ messenger RNA expression, as assessed using macro-dissected tumor tissue RNA measurements at baseline. Secondary end points included PFS and OS in the intention-to-treat population comprising all enrolled patients (including those with EGFR or $A L K$ genomic alterations), PFS as determined at an independent review facility, investigator-assessed PFS in the PD-L1 expression subgroups, the objective response rate, and the duration of response among patients with an objective response.

The WT population comprised 1,040 patients $(86.5 \%)$, and 445 of them (42.8\%) had high Teff gene-signature expressions. At the time of data cutoff, the PFS of the WT population was significantly longer in the ABCP group than in the BCP group $[8.3$ vs. 6.8 months; hazard ratio (HR) 0.62 ; $95 \%$ confidence interval $(\mathrm{CI}), 0.52-0.74$; $\mathrm{P}<0.001]$. In the Teff-high WT population, the PFS was also significantly longer in the ABCP group than in the BCP group (11.3 vs. 6.8 months; HR 0.51; 95\% CI, $0.38-0.68 ; \mathrm{P}<0.001)$. The PFS among patients with $E G F R$ mutations or $A L K$ rearrangements increased with $\mathrm{ABCP}$ compared with that with BCP (9.7 vs. 6.1 months; HR, 0.59; 95\% CI, 0.37-0.94). Additionally, in all intention-to-treat patients (including those with EGFR mutations or ALK rearrangements), the PFS increased with $\mathrm{ABCP}$ than with $\mathrm{BCP}$ at 8.3 vs. 6.8 months (HR 0.61; 95\% CI, 0.52-0.72). Prolonged PFSs were observed regardless of PD-L1 status and expression of Teff gene signature. One benefit of PFS was observed with ABCP in key clinical and biomarker subgroups: in patients with liver metastasis (7.4 vs. 4.9 months; HR 0.42 ; $95 \%$ CI, 0.26-0.66) and in patients with KRAS mutations (8.1 vs. 5.8 months; HR 0.50; 95\% CI, 0.29-0.84).

At the time of the interim analysis, the OS in the WT population was significantly longer in the $\mathrm{ABCP}$ group than in the BCP group (19.2 vs. 14.7 months; HR 0.78; 95\% CI, 0.64-0.96; $\mathrm{P}=0.02$ ). The investigator-assessed unconfirmed objective response rates in the WT were $63.5 \%$ in the $\mathrm{ABCP}$ group and $48.0 \%$ in the BCP. In the $\mathrm{WT}$ population, the median response durations were 9.0 and 5.7 months in the $\mathrm{ABCP}$ and $\mathrm{BCP}$ groups, respectively; in the Teff-high WT population, these were 11.2 and 5.7 months in the $\mathrm{ABCP}$ and $\mathrm{BCP}$ groups, respectively.

Grade 1 or 2 adverse events occurred in $35.9 \%$ and $45.4 \%$ of patients in the ABCP and BCP groups, respectively. The most commonly observed treatmentrelated adverse events (grade 3 or 4) were neutropenia and hypertension. The incidences of rash, stomatitis, febrile neutropenia, and hemoptysis were higher by $<10 \%$ among patients in the $\mathrm{ABCP}$ group than among those in the BCP group. Deaths from treatment occurred in $11(2.8 \%)$ and $9(2.3 \%)$ patients in the ABCP and BCP groups, respectively. Five deaths in the ABCP group were due to pulmonary hemorrhage or hemoptysis. In all, 77.4\% of the immune-related adverse events observed in the $\mathrm{ABCP}$ group belonged to grade 1 or 2 , and none of these belonged to grade 5 . The most common observed immune-related adverse events included rash, hepatitis, hypothyroidism, hyperthyroidism, pneumonitis, and colitis.

The study was the first phase III trial that reported on chemotherapy combination, immunotherapy, and antiangiogenic treatment as first-line treatment for advanced non-squamous NSCLC. The results demonstrated significantly improved PFS and OS with the inclusion of atezolizumab in the $\mathrm{BCP}$ regimen as a firstline treatment for non-squamous metastatic NSCLC. Compared with the results of the ECOG4599 (14), a trial of paclitaxel and carboplatin or of paclitaxel and carboplatin 


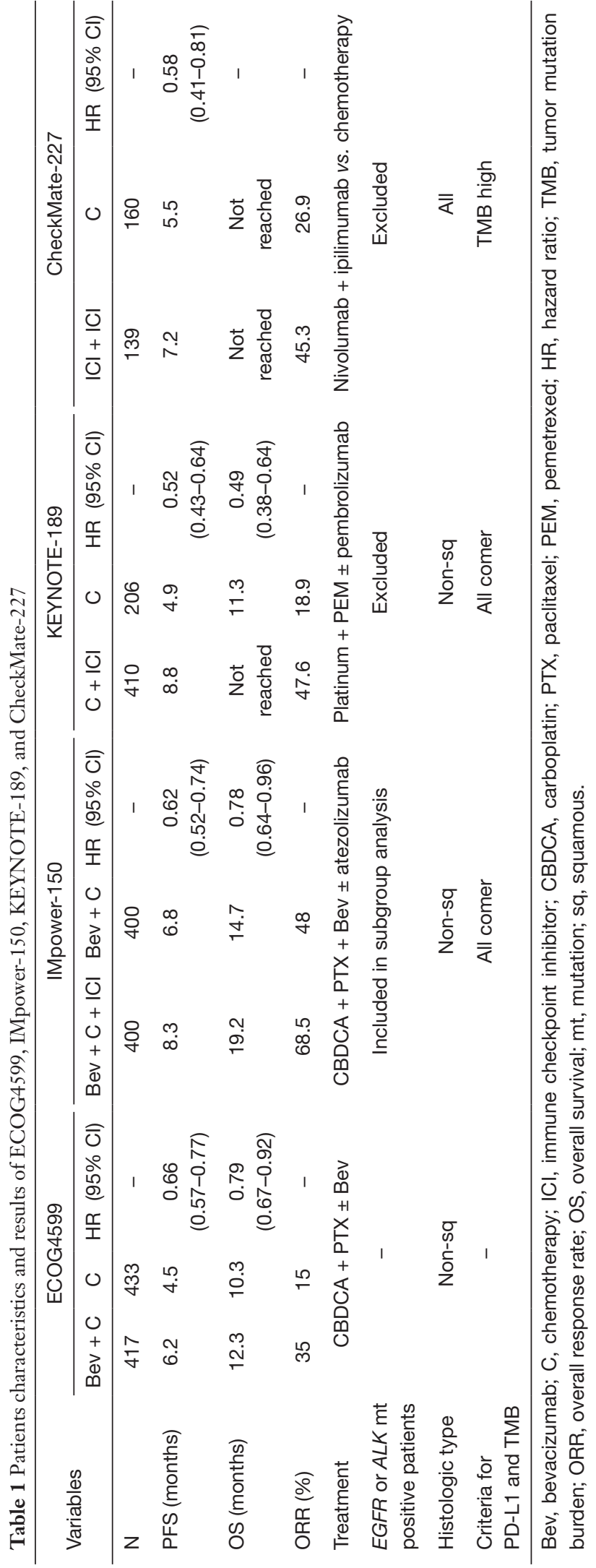

with bevacizumab, the superiority of the $\mathrm{ABCP}$ treatment is apparent by comparing the OS, PFS, and ORR (Table 1). Benefits with respect to OS, PFS, and ORR were found in all subgroups examined, including those that had a PD-L1 tumor proportion score of $<1 \%$. These findings in unselected patients with metastatic NSCLC are particularly important because the effect of the PD-1 inhibitor monotherapy has only been proven in patients with positive PD-L1 expression. The inclusion of pembrolizumab to pemetrexed and a platinum-based drug (standard chemotherapy) has been shown to result in significantly longer OS and PFS than chemotherapy alone in patients with untreated metastatic non-squamous NSCLC and any PD-L1 immunohistochemistry status without EGFR or ALK mutations (KEYNOTE-189) (15). The combination of ICI treatment, nivolumab plus ipilimumab, was also shown to result in significantly longer PFS than that of chemotherapy among patients with NSCLC and a high tumor mutation burden (but without EGFR and $A L K$ mutations) (CheckMate-227) (16). The PFSs in these three trials were similar. Each trial was conducted on the basis of the different criteria, and the best treatment in selected patients is still unknown. In all, the results so far demonstrate the success of the ICI combination therapy for NSCLC, although the IMpower-150 trial only showed benefit in patients with EGFR and $A L K$ genetic alterations. In some clinical trials, retrospective, and metaanalysis studies, the use of PD-1 or PD-L1 inhibitors as monotherapy has shown relatively low efficacy in patients with $E G F R$ or $A L K$ genetic alterations $(1,3,4,17,18)$. In particular, atezolizumab monotherapy was not effective for patients with $E G F R$ or $K-R A S$ genetic alterations in a phase III trial of atezolizumab versus docetaxel in patients with previously treated NSCLC (OAK trial) (4); therefore, the combination of atezolizumab and bevacizumab with chemotherapy may correct this result producing an additive effect useful against $E G F R$ and $A L K$ gene alterations. Another report showed that T790M-negative patients with NSCLC and positive-EGFR mutations are more likely to benefit from nivolumab than the T790M-positive counterparts after disease progression with EGFR-TKI (19), but the proportion of T790M-negative patients with positive EGFR mutations after EGFR-TKI treatment was unknown in the IMpower-150 study. Other clinical trials are being conducted for patients with EGFR mutations after EGFR-TKI treatment (Table 2) and the partial results may suggest the new combination immunotherapy which is effective for NSCLC patients with EGFR mutations. 
Table 2 Ongoing trials of combination immunotherapy after treatment with EGFR-TKI in NSCLC

\begin{tabular}{|c|c|c|c|c|c|}
\hline NCT & $\begin{array}{l}\text { Study } \\
\text { phase }\end{array}$ & $\mathrm{ICl}$ & $\begin{array}{l}\text { Gene } \\
\text { mutation }\end{array}$ & T790M & Treatment sequence \\
\hline NCT03091491 & II & Nivo, Ipi & $E G F R$ & Allowed & EGFR-TKI, no more than 1 line chemo $\rightarrow$ Nivo or Nivo/Ipi \\
\hline NCT03242915 & II & Pembro & EGFR, ALK & Not described & EGFR-TKI $\rightarrow$ CBDCA + PEM + Pembro \\
\hline NCT03256136 & II & Nivo, Ipi & EGFR, ALK & Not described & $\begin{array}{l}\text { Cohort A: } 3^{\text {rd }} \text { generation EGFR-TKI } \rightarrow \text { CBDCA + PEM + Nivo } \\
\text { Cohort C: } 3^{\text {rd }} \text { generation EGFR-TKI } \rightarrow \text { platinum-based } \\
\text { chemotherapy } \rightarrow \text { Nivo/lpi }\end{array}$ \\
\hline $\begin{array}{l}\text { NCT02454933 } \\
\text { (CAURAL) }\end{array}$ & III & Durva & EGFR & Positive & $\begin{array}{l}\text { EGFR-TKI } \rightarrow \text { additional anti-cancer treatment is permitted } \\
\rightarrow \text { osimertinib or Durva/osimertinib }\end{array}$ \\
\hline $\begin{array}{l}\text { NCT02864251 } \\
\text { (CheckMate-722) }\end{array}$ & III & Nivo, Ipi & EGFR & Negative & $\begin{array}{l}\text { EGFR-TKI } \rightarrow \text { platinum + PEM or platinum + PEM + Nivo or } \\
\text { Nivo/Ipi }\end{array}$ \\
\hline
\end{tabular}

TKI, tyrosine kinase inhibitor; Nivo, nivolumab; Pembro, pembrolizumab; Ipi, ipilimumab; Durva, durvalumab; CBDCA, carboplatin; PEM, pemetrexed; ICI, immune checkpoint inhibitor.

Detailed and extensive preclinical and clinical rationale data support the hypothesis that anti-vascular endothelial growth factor (VEGF) may act synergistically with immunotherapy benefiting patients (20). VEGF suppresses tumor-directed immune responses and promotes angiogenesis. Altering these effects in the tumor microenvironment by inhibiting angiogenesis is an appealing combination strategy for ICIs. Combination therapy promotes antitumor activity and has beneficial effects on the host antitumor immune response. Because VEGF signaling attenuates the antitumor response through the inhibition of lymphocyte adhesion (21) and the regulation of immune cells $(22,23)$, antiangiogenic agents may bring about stimulation of the immune response and enhancement of the efficacy of immunotherapies.

To adapt the combination therapy of bevacizumab, atezolizumab, and chemotherapy for NSCLC patients, thrombosis, bleeding, gross hemoptysis, and hypertension need to be ruled out. Regardless of the existence of these restrictions and based on the IMpower-150 results, the ABCP combination therapy may be especially useful for patients who have $E G F R$ or $A L K$ mutations or liver metastases. The NSCLC patients who have pleural fluid or small brain metastases may be also eligible to use this regimen because of the efficacy of bevacizumab on them $(24,25)$. Moreover, according to its better ORR compared with others (Table 1), this regimen may be also adequate for patients requiring tumor volume reduction, i.e., for the treatment of trachea stenosis and superior vena cava syndrome or to prevent rupture of liver metastases.

In conclusion, the Impower-150 study showed that addition of atezolizumab to the regimen of bevacizumab plus chemotherapy as first-line treatment for non-squamous metastatic NSCLC resulted in significantly improved PFS and OS. Regardless of the expression of PD-L1 or the presence of a T-effector gene signature, the combination therapy proved beneficial. In addition, the clinical benefits were clearly observed at baseline in key subgroups of patients with $E G F R$ and $A L K$ genomic alterations and liver metastases. The trial showed that combining chemotherapy and immunotherapy helps avoid the requirement for strict patient selection (based on the presence of biomarkers); thus, the strategy can potentially benefit large patient populations with advanced NSCLC without encountering practical difficulties involving biomarker testing. Different ICI combination therapies have shown promising results, and the IMpower-150 showed the efficacy of atezolizumab plus bevacizumab in combination as one chemotherapy regimen. Each combination therapy has its own features, and the choice of the best combination therapy needs to be based on its features and the patients' characteristics (their condition and gene profiling).

\section{Acknowledgments}

Funding: None. 


\section{Footnote}

Provenance and Peer Review: This article was commissioned and reviewed by the Section Editor Wei Xu (Division of Respiratory Disease, Department of Geriatrics, the First Affiliated Hospital of Nanjing Medical University, Nanjing, China).

Conflicts of Interest: Both authors have completed the ICMJE uniform disclosure form (available at http://dx.doi. org/10.21037/tcr.2018.08.14). T Hida has received grants and personal fees from AstraZeneca, Ono Pharmaceutical, Chugai Pharmaceutical, Eli Lilly, Novartis, Taiho Pharmaceutical, Nippon Boehringer Ingelheim, Pfizer, Bristol-Meyers Squibb, Clovis Oncology, MSD, and Kissei and grants from Eisai, Takeda Pharmaceutical, Dainippon Sumitomo Pharma, Abbvie, Merck Serono, Kyowa Hakko Kirin, Daiichi Sankyo, Astellas, Ignyta, and Servier. T Uemura has no conflicts of interest to declare.

Ethical Statement: The authors are accountable for all aspects of the work in ensuring that questions related to the accuracy or integrity of any part of the work are appropriately investigated and resolved.

Open Access Statement: This is an Open Access article distributed in accordance with the Creative Commons Attribution-NonCommercial-NoDerivs 4.0 International License (CC BY-NC-ND 4.0), which permits the noncommercial replication and distribution of the article with the strict proviso that no changes or edits are made and the original work is properly cited (including links to both the formal publication through the relevant DOI and the license). See: https://creativecommons.org/licenses/by-nc-nd/4.0/.

\section{References}

1. Borghaei H, Paz-Ares L, Horn L, et al. Nivolumab versus Docetaxel in Advanced Nonsquamous Non-Small-Cell Lung Cancer. N Engl J Med 2015;373:1627-39.

2. Brahmer J, Reckamp KL, Baas P, et al. Nivolumab versus Docetaxel in Advanced Squamous-Cell Non-Small-Cell Lung Cancer. N Engl J Med 2015;373:123-35.

3. Herbst RS, Baas P, Kim DW, et al. Pembrolizumab versus docetaxel for previously treated, PD-L1-positive, advanced non-small-cell lung cancer (KEYNOTE-010): a randomised controlled trial. Lancet 2016;387:1540-50.

4. Rittmeyer A, Barlesi F, Waterkamp D, et al. Atezolizumab versus docetaxel in patients with previously treated non-small-cell lung cancer (OAK): a phase 3, openlabel, multicentre randomised controlled trial. Lancet 2017;389:255-65.

5. Garon EB, Rizvi NA, Hui R, et al. Pembrolizumab for the treatment of non-small-cell lung cancer. N Engl J Med 2015;372:2018-28.

6. Antonia SJ, Villegas A, Daniel D, et al. Durvalumab after Chemoradiotherapy in Stage III Non-Small-Cell Lung Cancer. N Engl J Med 2017;377:1919-29.

7. Apetoh L, Ladoire S, Coukos G, et al. Combining immunotherapy and anticancer agents: the right path to achieve cancer cure? Ann Oncol 2015;26:1813-23.

8. Galluzzi L, Buque A, Kepp O, et al. Immunological Effects of Conventional Chemotherapy and Targeted Anticancer Agents. Cancer Cell 2015;28:690-714.

9. Zitvogel L, Galluzzi L, Smyth MJ, et al. Mechanism of action of conventional and targeted anticancer therapies: reinstating immunosurveillance. Immunity 2013;39:74-88.

10. Peng J, Hamanishi J, Matsumura N, et al. Chemotherapy Induces Programmed Cell Death-Ligand 1

Overexpression via the Nuclear Factor-kappaB to Foster an Immunosuppressive Tumor Microenvironment in Ovarian Cancer. Cancer Res 2015;75:5034-45.

11. Zhang $\mathrm{P}, \mathrm{Ma} \mathrm{Y}, \mathrm{Lv} \mathrm{C}$, et al. Upregulation of programmed cell death ligand 1 promotes resistance response in nonsmall-cell lung cancer patients treated with neo-adjuvant chemotherapy. Cancer Sci 2016;107:1563-71.

12. Langer CJ, Gadgeel SM, Borghaei H, et al. Carboplatin and pemetrexed with or without pembrolizumab for advanced, non-squamous non-small-cell lung cancer: a randomised, phase 2 cohort of the open-label KEYNOTE-021 study. Lancet Oncol 2016;17:1497-508.

13. Socinski MA, Jotte RM, Cappuzzo F, et al. Atezolizumab for First-Line Treatment of Metastatic Nonsquamous NSCLC. N Engl J Med 2018;378:2288-301.

14. Sandler A, Gray R, Perry MC, et al. Paclitaxel-carboplatin alone or with bevacizumab for non-small-cell lung cancer. N Engl J Med 2006;355:2542-50.

15. Gandhi L, Rodriguez-Abreu D, Gadgeel S, et al. Pembrolizumab plus Chemotherapy in Metastatic NonSmall-Cell Lung Cancer. N Engl J Med 2018;378:2078-92.

16. Hellmann MD, Ciuleanu TE, Pluzanski A, et al. Nivolumab plus Ipilimumab in Lung Cancer with a High Tumor Mutational Burden. N Engl J Med 2018;378:2093-104.

17. Gainor JF, Shaw AT, Sequist LV, et al. EGFR Mutations and ALK Rearrangements Are Associated with Low Response Rates to PD-1 Pathway Blockade in Non-Small 
Cell Lung Cancer: A Retrospective Analysis. Clin Cancer Res 2016;22:4585-93.

18. Lee CK, Man J, Lord S, et al. Checkpoint Inhibitors in Metastatic EGFR-Mutated Non-Small Cell Lung Cancer-A Meta-Analysis. J Thorac Oncol 2017;12:403-7.

19. Haratani K, Hayashi H, Tanaka T, et al. Tumor immune microenvironment and nivolumab efficacy in EGFR mutation-positive non-small-cell lung cancer based on T790M status after disease progression during EGFRTKI treatment. Ann Oncol 2017;28:1532-9.

20. Manegold C, Dingemans AC, Gray JE, et al. The Potential of Combined Immunotherapy and Antiangiogenesis for the Synergistic Treatment of Advanced NSCLC. J Thorac Oncol 2017;12:194-207.

21. Bouzin C, Brouet A, De Vriese J, et al. Effects of vascular endothelial growth factor on the lymphocyte-endothelium interactions: identification of caveolin-1 and nitric oxide as control points of endothelial cell anergy. J Immunol

Cite this article as: Uemura T, Hida T. Additive effects of atezolizumab and bevacizumab plus chemotherapy for patients with non-small cell lung cancer regardless of presence of EGFR mutations, ALK rearrangements, or PD-L1 expression. Transl Cancer Res 2018;7(Suppl 7):S796-S801. doi: 10.21037/ tcr.2018.08.14
2007;178:1505-11.

22. Gabrilovich DI, Chen HL, Girgis KR, et al. Production of vascular endothelial growth factor by human tumors inhibits the functional maturation of dendritic cells. Nat Med 1996;2:1096-103.

23. Ohm JE, Gabrilovich DI, Sempowski GD, et al. VEGF inhibits T-cell development and may contribute to tumorinduced immune suppression. Blood 2003;101:4878-86.

24. Besse B, Le Moulec S, Mazieres J, et al. Bevacizumab in Patients with Nonsquamous Non-Small Cell Lung Cancer and Asymptomatic, Untreated Brain Metastases (BRAIN): A Nonrandomized, Phase II Study. Clin Cancer Res 2015;21:1896-903.

25. Usui K, Sugawara S, Nishitsuji M, et al. A phase II study of bevacizumab with carboplatin-pemetrexed in nonsquamous non-small cell lung carcinoma patients with malignant pleural effusions: North East Japan Study Group Trial NEJ013A. Lung Cancer 2016;99:131-6. 